\title{
Effect of Local Implantation of Composite Biomaterial Loaded with Vascular Endothelial Growth Factor in Healing of Critical-Sized Mandibular Defect - A Pilot Study
}

\author{
Hamid H. Enezei ${ }^{1,2 *}$, Azlina A $^{1}$, Khamis MF ${ }^{1}$, Rahman RA' ${ }^{1}$ Abdul Razak NH$^{1}$, Mutum SS ${ }^{3}$ and \\ Samsudin $\mathbf{A R}^{4}$ \\ ${ }^{1}$ School of Dental Sciences, University Sains Malaysia Health Campus, 16150 Kelantan, Malaysia \\ ${ }^{2}$ College of Dentistry, Anbar University, Oral and Maxillofacial Surg. Department Ramadi, Iraq \\ ${ }^{3}$ School of Medical Sciences, University Sains Malaysia Health Campus, 16150 Kelantan, Malaysia \\ ${ }^{4}$ College of Dentistry, University of Sharjah, Sharjah, United Arab Emirates
}

Received: June 06,2014; Accepted: July 12,2014; Published: July 18,2014

*Corresponding author: Hamid Hammad Enezei, School of Dental Sciences, University Sains Malaysia Health Campus, 16150 Kelantan, Malaysia, Tel: +6-0179-295-821; E-mail: drhamed2000@yahoo.com

\begin{abstract}
This study was aimed to establish the optimal effective concentration of VEGF in bone regeneration in vivo. Four male adult white New Zealand rabbits were equally divided into two experimental groups, A and B, which were also used as control. One circular critical size defect 8-mm $\emptyset$ was created bilaterally in the mandibular body area of each rabbit. Group A received composite BCP/FS (Biphasic Calcium Phosphate/Fibrinogen Solution) with 250 ng/ml VEGF (Vascular Endothelial Growth Factor) on one side, and the control side received BCP/FS only. Group B received composite $\mathrm{BCP} / \mathrm{FS}$ with $500 \mathrm{ng} / \mathrm{ml}$ VEGF on one side, and the control side received only BCP/FS. 4 weeks later, group $B$ showed significant increase in bone formation compared to that of group $\mathrm{A}$, and least bone formation in the control.
\end{abstract}

Keywords: Critical size bone defects; Composite bone graft; Vascular endothelial growth factor

\section{Introduction}

In human beings, one of the most challenging problems in oral and maxillofacial surgery is the repair of Critical-Sized Bone Defects (CSBDs) in the mandible that needs attention Tai et al. [1] demonstrated that treatment of CSBDs using Mesenchymal Stem Cells (MSCs) with suitable biodegradable scaffolds is feasible [1]. However, this approach is not effective for CSBDs due to inadequate vascularization [2]. In order to encourage the vascularization process, the cytokines like Vascular Endothelial Growth Factor (VEGF) act as strong promoter agent of neovascularization for effective coupling of angiogenesis and osteogenesis of bone substitutes. The efficacy of VEGF in bone healing process depends on proper dosage, appropriate delivery system that has adequate release kinetics and high biological activity [3]. Thus, this study was aimed to establish the optimal effective concentration of VEGF in vivo. However, the VEGF has some drawbacks. In high dose administration it has negative effects on healing process [3]. Furthermore, its half- life is short when injected into the tissues $[4,5]$. Considering the drawbacks and challenge from CSBD, we hypothesized that enriched Biphasic Calcium Phosphate (BCP) with VEGF and Fibrin Sealant (FS) can act as a scaffold with continuous release of low concentration of VEGF into the area of healing to promote neovascularization.

\section{Work Methodology}

All animal procedures were conducted under a protocol approved by the Universiti Sains Malaysia Animal Ethics Committee USM (AECUSM) USM/2012/1-July-2012/1July-2014), No. of Animal Ethics Approval/USM/Animal Ethics Approval/2012/(81)/(419). FS was purchased from Baxter Healthcare Corporation, Westlake Village, CA-91362 USA, US License No. 140. VEGF was purchased from GIBCO, USA. Macroporous Biphasic Calcium Phosphate (MBCP) ceramic granules were synthesized in Ceramic Laboratory, School of Materials and Mineral Resources, Engineering Campus, Universiti Sains Malaysia (USM). The preparation of FS and VEGF was in accordance with the manufacturer's recommendations. Four male white New Zealand rabbits (Oryctolagus cuniculus), aged between 5 and 6 months, and with a mean weight $2.63 \mathrm{~kg} \pm$ $0.35 \mathrm{~kg}$ were divided into two experimental groups A and B. The experimental rabbits were also used as their own control (figure 1 and 2).

Rabbits were sedated before surgery with ketamine-xylazine IM injection, ketamine $35 \mathrm{mg} / \mathrm{kg}$ and xylazine $5 \mathrm{mg} / \mathrm{kg}$ [6]. Anaesthesia was induced and maintained by administration of $1 \%-3 \%$ sevoflurane mixed with oxygen via a face mask. Rabbits were put in lateral position during surgery, and the heart rate, 
respiratory rate, pulse oximetry, and temperature of the animals were monitored. Before the operation, the area was shaved and prepared with betadine, and a subcutaneous injection of $0.5 \%$ lidocaine with 1:200,000 epinephrine was given in the line of incision (incision line block). One circular critical-sized trephine defect 8-mm $\emptyset$ was created bilaterally in the mandibular body area (the premolar/molar region) removing only the lateral bony cortex, trabecular bone, and tooth roots (partial thickness).

Group A received composite BCP/FS loaded with $250 \mathrm{ng} / \mathrm{ml}$ VEGF, while the other side received only composite without VEGF (control). Group B received composite BCP/FS loaded with 500 $\mathrm{ng} / \mathrm{ml}$ while the other side received only composite without VEGF (control). The periosteal flap was repositioned and the wound closed in layers using 4-0 coated vicryl suture. Postoperatively, each animal was observed closely by a veterinary technician and operator until it regained consciousness, at which time, it was transferred to the individual cages. Subcutaneous infusion of $5 \%$ glucose water was given with oral orange juice for the initial feeding. Thereafter, the animals were fed normally. In each animal the wound site was debrided daily and cleaned with normal saline and povidone iodine for 7 days. Neomycin local antibiotic cream was applied daily for the first week to the incision site. Intramuscular injection of Baytril (Enrofloxacin), $10 \mathrm{mg} / \mathrm{kg} /$ day once a day for 7 days followed. Tramadol hydrochloride $2 \mathrm{mg} / \mathrm{kg}$ once daily was given for pain relief for 7 days. The weight of the rabbits was recorded daily and any change in the dietary habits or activity was monitored closely. The animals were euthanized at 4 weeks after surgery with an intravenous injection of overdose sodium pentobarbitone $(100 \mathrm{ml} / \mathrm{kg})$ and the tissues from the defect areas were collected. The samples were fixed in $4 \%$ paraformaldehyde for $12 \mathrm{~h}$ at room temperature. All samples were scanned for bone formation within the defect site using a Scanco system ( $\mu$ CT 80, Scanco Medical Switzerland). After the $\mu \mathrm{CT}$ scanning, all tissue samples were decalcified in $10 \%$ formic acid for tissue processing. All sections were cut and stained with haematoxylin and eosin (H\&E) for histomorphometric analysis (Carl Zeiss image analysis system MMI 0684).

\section{Results}

The results of the serial postoperative examination revealed presence of radiological and histological evidence of critical size bone healing in all treatment groups.

\section{$\mu \mathrm{CT}$ evaluation}

The percentage of remaining residual composite was significantly lower at 4 weeks in BCP/FS loaded with the 500 $\mathrm{ng} / \mathrm{ml} \mathrm{VEGF}$ than in the $250 \mathrm{ng} / \mathrm{ml}$ of VEGF group as a result of composite utilization. $\mu \mathrm{CT}$ picture shows radio-lucent area which is gradually reduced in size and is replaced by opacities encroaching from the periphery to the centre of the bone defect with time according to the rate of new bone formation after surgery (Figure3). Group B- BCP/FS loaded with $500 \mathrm{ng} / \mathrm{ml}$ showed more opacities than group $\mathrm{A}$. The bone regeneration was increasingly radio-opaque. A clear boundary with the host bone was observed and different radio-opacities from the periphery
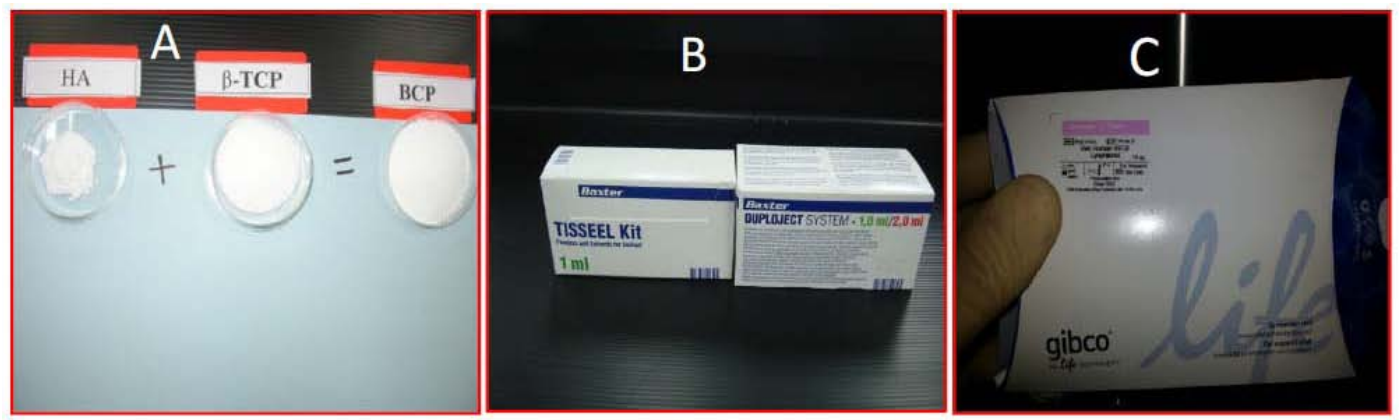

Figure 1: $\mathrm{A}=\mathrm{BCP}$ granules, $\mathrm{B}=\mathrm{FS}, \mathrm{C}=\mathrm{VEGF}$.
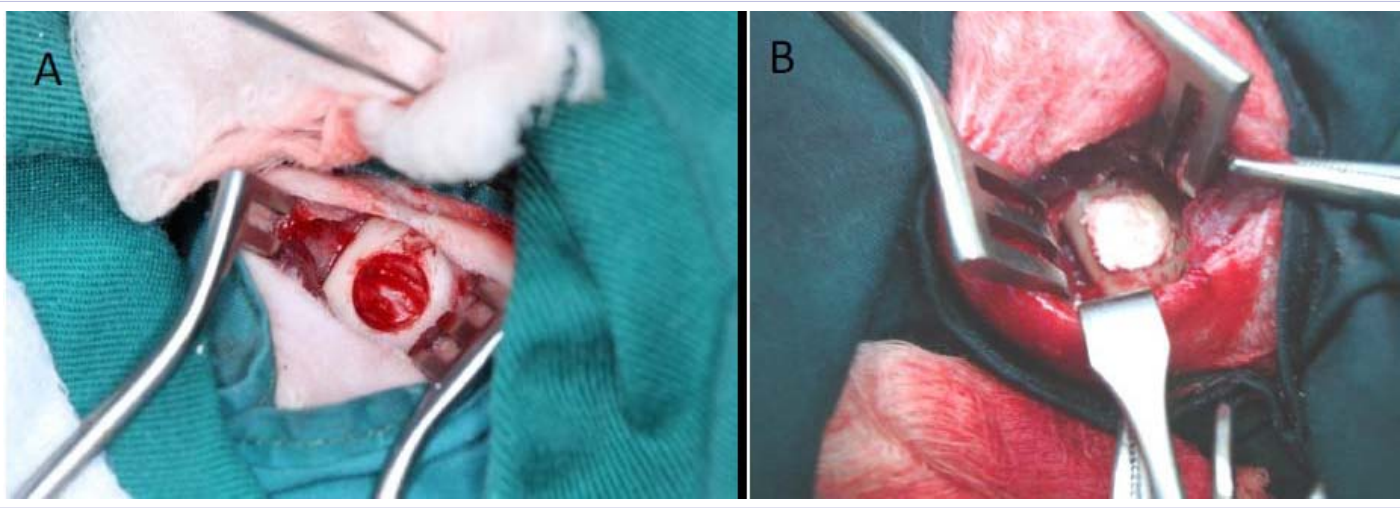

Figure 2: A: CSBD before implantation of composite bone graft. B: CSBD after implantation of composite bone graft.

Citation: Enezei HH, Azlina A, Khamis MF, Rahman RA, Abdul Razak NH, et al. (2014) Effect of Local Implantation of Composite Biomaterial Loaded with Vascular Endothelial Growth Factor in Healing of Critical-Sized Mandibular Defect - A Pilot Study. J Dent 


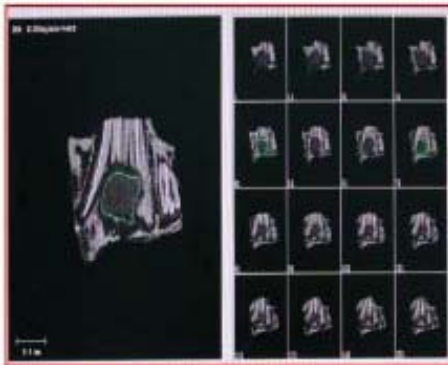

A

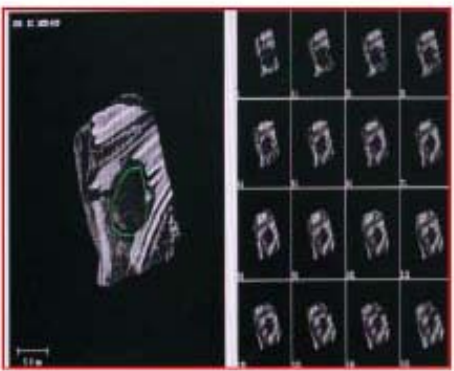

control

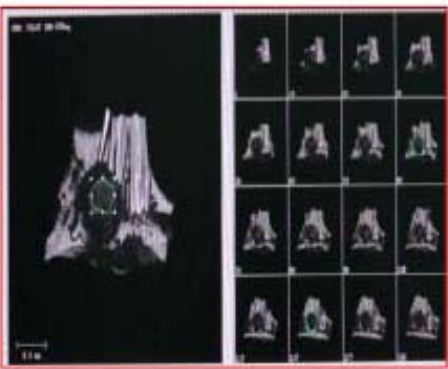

B

Figure 3: $\mu$ CT pictures shows a radiolucent zone (halo) surrounding the graft material with minimal size in group B compared to group A and control.

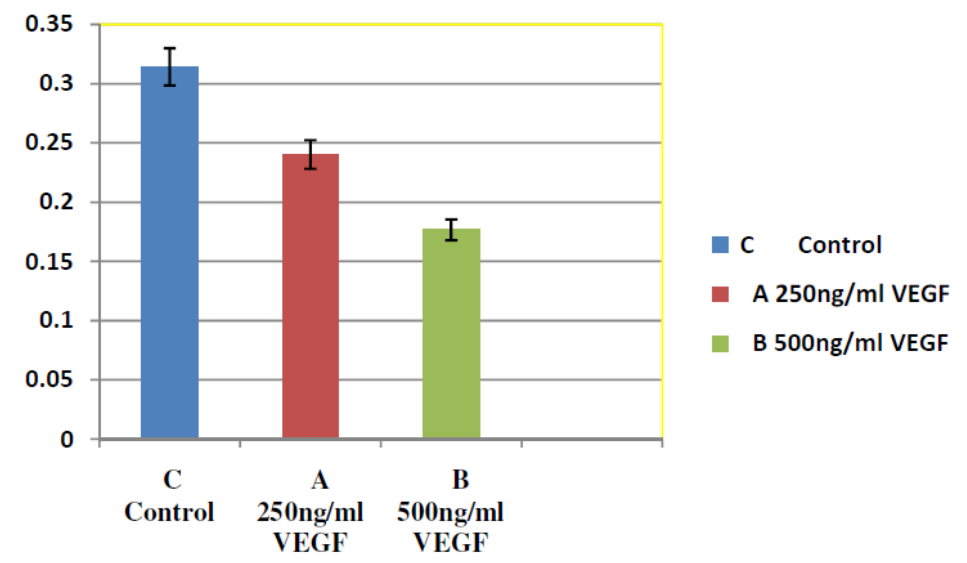

Figure 4: Mean difference of remaining residual composite (radio-lucent) in group A and B in relation to control group which was measured by $\mu$ CT less radiolucency mean more new bone formation which appears clear in group B.

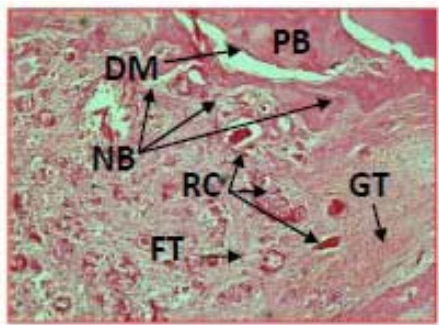

A

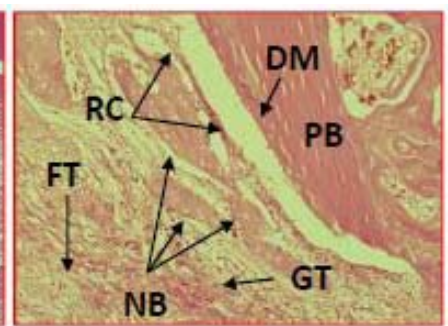

control

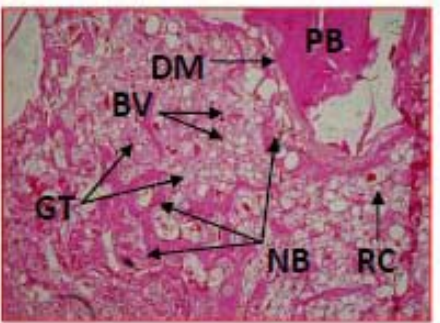

B

Figure 5: Histological presentation at 4 weeks of CSBDs healing in rabbit's mandible in control group, group A and group B (H\&E 40x). Group A shows less new bone formed, with some fibrous tissue, more Residual Composite (RC) and minimal granulation tissue. Group B shows more scattered new Blood Vessels (BV) from Defect Margin (DM), more Granulation Tissue (GT), with minimal (RC), marked a New Bone formed (NB) in thick layers and Pre-Existing bone (PB) appear clearly. In control group a limited amount of new bone formation, more Fibrous tissue (FT), inflammatory infiltration but maximum residual composite seen.

toward the centre of the defect were visible. The amount of newly formed bone to the total implant area in composite loaded with $500 \mathrm{ng} / \mathrm{ml}$ group B was significantly greater than that in the composite loaded with $250 \mathrm{ng} / \mathrm{ml}$ group A and control. Mean difference of residual composite in group B was less than the mean difference in group A while mean difference of residual composite was highest in control group (Figure 4).

\section{Histological and Histomorphometrical findings}

Histological examination of H\&E stained slides demonstrated that more new bone formation occurred in the defect of group $\mathrm{B}$ compared to the defect in group A and control. It was obvious that composite loaded $500 \mathrm{ng} / \mathrm{ml}$ VEGF induced more new bone formation than composite loaded with $250 \mathrm{ng} / \mathrm{ml}$ four weeks after implantation. Only little new bone was formed in the composite 


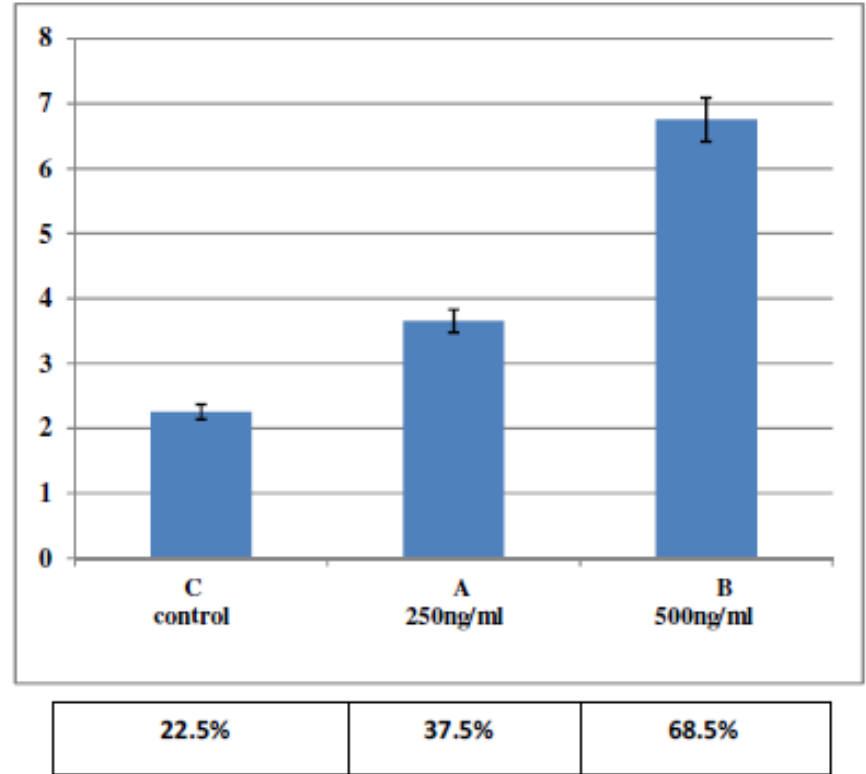

Figure 6: Histomorphometrical results show the mean difference of groups A and B compared with control. Extensive new bone formation in group $\mathrm{B}$ than in group A and control.

loaded with $250 \mathrm{ng} / \mathrm{ml}$ group. Four weeks after implantation, most of the composite biomaterial was degraded with more blood vessels at the periphery and the centre of the defect, the new bone formed filled most parts of the defect in group B. However, the defect in group A showed more fibrous tissue than in group B. It was obvious that composite loaded with $500 \mathrm{ng} / \mathrm{ml}$ VEGF induced more new bone formation than composite loaded with $250 \mathrm{ng} / \mathrm{ml}$ (Figure 5). Histomorphometrical analysis confirm the histological findings that the amount of newly formed bone to the total implant area in composite loaded $500 \mathrm{ng} / \mathrm{ml}$ group B was significantly greater than that in the composite loaded $250 \mathrm{ng} / \mathrm{ml}$ group A and control. For experiment group the mean difference of group B was higher than the mean difference of group A and control group meaning that the amount of new bone formation in group B with composite loaded $500 \mathrm{ng} / \mathrm{ml}$ VEGF was higher than the amount of bone formation in group A implanted with composite loaded with $250 \mathrm{ng} / \mathrm{ml}$ VEGF. Mean difference of group A and B was higher than the mean of the control group implanted with composite only. Percentage of newly formed bone in control group, composite BCP/FS loaded with $250 \mathrm{ng} / \mathrm{ml}$ VEGF and composite BCP/FS loaded with $500 \mathrm{ng} / \mathrm{ml}$ were $22.5 \%$, $37.5 \%$ and $68.5 \%$ respectively as shown in (Figure 6).

\section{Discussion}

Existing treatment of CSBDs using autogenous and allograft procedures are limited and often associated with undesirable outcomes [7]. Bone xenografts are now considered to be unsuitable to use as bone graft due to a potential risk of contamination with viral and bacterial infections, immunogenicity, and finally host rejection [8]. Composite biomaterials containing calcium phosphate and polymers (natural or synthetic) have been developed to address these limitations [9]. FS is a candidate polymer for use in fabricating composite biomaterials and delivery vehicle of growth factor for bone regeneration. Numerous data showed that effects of growth factors on both angiogenesis and bone formation are dose-dependent. The required dose of growth factor varied with the mode of administration and carrier type. In general, localized and sustained release of growth factors allows for lower effective dosage than a single injection [3]. VEGF has been identified to have positive effects on osteogenesis and angiogenesis [10,11]. Appropriate dosage and release profiles are very important for optimized biomolecule delivery. In addition, the application of excessive dose may provoke adverse effects or even toxic reactions, such as inhibition of blood vessels and bone formation [3]. Similarly, slow and sustained release of VEGF can produce well-functioning blood vessels, whereas uncontrolled release of VEGF leads to malformed and non functional blood vessels [12]. Therefore, optimizing and controlling the release of VEGF in vitro and in vivo is one of the primary concerns in bone tissue engineering.

Our results are consistent with Hobson's work [13] that VEGF at $500 \mathrm{ng} / \mathrm{ml}$ generally stimulates a preferable vascularization. However, very little is known about potential mechanisms that may confine the induction of VEGF within an appropriate magnitude for proper angiogenesis. Because a precise dose of VEGF is critical for vascularization, disruption of such mechanisms may contribute to pathological angiogenesis [11].

In group B, the regeneration within the defect, characterized by increasing radio-opacity while there is a gradual reduction of the radiolucent zone (halo) surrounding the graft material, indicates more efficient graft resorption when compared to group A and control. The absence of such a radiolucent zone in defect treatment may be indicative of improved new bone formation mediated by the novel bone substitute material. The radiologically detectable sclerotic defect margin present 
in the present case as well as the decrease in radiolucency can be explained by a summation effect of new bone formation and the bone substitute material still in place. No adverse effect or biological complications has occurred that can be attributed to the biomaterial. Composite in group B showed more abundant blood circulation in the bone defect areas than samples from group A and control because of the physiological function of VEGF as clarified by Yang et al. [14]. This could explain why composite in group B had a higher degradation rate than those of group A and control. In the present study, there was no cartilaginous or fibrocartilaginous tissue in the newly formed bone, and the type of ossification was described as intramembranous in origin [15].

\section{Conclusions}

The combination of BCP/FS loaded with $500 \mathrm{ng} / \mathrm{ml}$ VEGF significantly accelerates bone healing in CSBDs in vivo. The results of the present study suggest that $\mathrm{BCP}$ and FS are suitable to act as scaffold in the treatment of CSBD and able to maintain sustained release of low concentration of VEGF.

\section{Acknowledgement}

The authors would like to thank Universiti Sains Malaysia for the financial support. We also express our gratitude to the staff of Craniofacial Biology Laboratory and the staff of animal house (ARASC) for their help.

\section{References}

1. Tai K, Pelled G, Sheyn D, Bershteyn A, Han L, Kallai I, et al. Nanobiomechanics of repair bone regenerated by genetically modified mesenchymal stem cells. Tissue Eng Part A. 2008; 14:1709-1720. doi: 10.1089/ten.tea.2007.0241

2. He X, Dziak R, Yuan X, Mao K, Genco R, Swihart M, et al. BMP2 Genetically Engineered MSCs and EPCs Promote Vascularized Bone Regeneration in Rat Critical-Sized Calvarial Bone Defects. PLoS One. 2013; 8(4):e60473. doi: 10.1371/journal.pone.0060473.

3. Bai Y, Yin G, Huang Z, Liao X, Chen X, Yao Y, et al. Localized delivery of growth factors for angiogenesis and bone formation in tissue engineering. Int Immunopharmacol. 2013; 16(2):214-23. doi: 10.1016/j.intimp.2013.04.001

4. Lee KY, Peters MC, Mooney DJ. Comparison of vascular endothelial growth factor and basic fibroblast growth factor on angiogenesis in
SCID mice. J Controlled Release. 2003; 87(1):49-56.

5. Wu C, Zhang Y, Zhou Y, Fan W, Xiao YA. comparative study of mesoporous glass/silk and non-mesoporous glass/silk scaffolds: Physiochemistry and in vivo osteogenesis. Acta Biomaterialia. 2012; $7(5): 2229-36$

6. Daculsi G, Uzel A, Weiss P, Goyenvall E, Aguado E. Developments in injectable multiphasic biomaterials. The performance of microporous biphasic calcium phosphate granules and hydrogels. J Mater Sci Mater Med. 2010; 21(3):855-61.

7. Mehta M, Schmidt-Bleek K, Duda GN, Mooney DJ. Biomaterial delivery of morphogens to mimic the natural healing cascade in bone. Adv Drug Deliv Rev. 2012; 64(12):1257-76. doi: 10.1016/j.addr.2012.05.006.

8. Yamano S, Haku K, Ishioka M, Lin TY, Hanatani S, Hanatani S, Dai J, et al. The Potential of Tissue Engineering and Regeneration for Craniofacial Bone. Dentistry. 2:136. doi:10.4172/2161-1122.1000136.

9. Osathanon T, Linnes ML, Rajachar RM, Ratner BD, Somerman MJ, Giachelli CM. Microporous nanofibrous fibrin-based scaffolds for bone tissue engineering. Biomaterials. 2008; 29(30):4091-9. doi: 10.1016/j.biomaterials.2008.06.030.

10. Bose S, Tarafder S. Calcium phosphate ceramic systems in growth factor and drug delivery for bone tissue engineering: A review. Acta Biomaterialia.2012; 8(4):1401-21. doi: 10.1016/j.actbio.2011.11.017.

11. Lu J \& Tang M. CTCF-dependent chromatin insulator as a built-in attenuator of angiogenesis. Transcription. 2012; 3(2):73-77. doi: $10.4161 /$ trns.19634

12. Kempen DH, Lu L, Heijink A, Hefferan TE, Creemers LB, Maran A, et al. Effect of local sequential VEGF and BMP-2 delivery on ectopic and orthotopic bone regeneration. Biomaterials. 2009; 30(14):281625. doi: 10.1016/j.biomaterials.2009.01.031.

13. Hobson MI. Increased vascularisation enhances axonal regeneration within an acellular nerve conduit. Ann R Coll Surg Engl. 2002; 84(1):47-53.

14. Yang P, Wang C, Shi Z, Huang X, Dang X, Li X, et al. rhVEGF 165 delivered in a porous beta-tricalcium phosphate scaffold accelerates bridging of critical-sized defects in rabbit radii. J Biomed Mater Res A. 2010; 92(2):626-40.

15. Sencimen M, Aydintug Y, Ortakoglu K, Karslioglu Y, Gunhan O, Gunaydin Y. Histomorphometrical analysis of new bone obtained by distraction osteogenesis and osteogenesis by periosteal distraction in rabbits. Int J Oral Maxillofac Surg. 2007; 36(3):235-242. 